\title{
Multi-sensor Data Fusion Based on Biased Estimation Method for Wind Speed Measurement
}

\author{
Xingjia Yao ${ }^{1, a^{*}}$, Yingbo Wang ${ }^{2, b}$, Wei $\mathrm{Wu}^{3, \mathrm{c}}$ and Jun Liao ${ }^{4, \mathrm{~d}}$ \\ ${ }^{1,2}$ Wind Energy Technology Institute, Shenyang University of Technology, Shenyang, China \\ ${ }^{3}$ CHINA Quality Certification Center (CQC) Shanghai Branch, Shanghai, China \\ ${ }^{4}$ DNVGL Group, Shanghai, China \\ axingjia@vip.163.com, ${ }^{b}$ keironwang@163.com, ${ }^{\mathrm{c}}$ wuwei@cqc.com.cn, ${ }^{\mathrm{d}}$ liao.jun@dnvgl.com
}

Keywords: wind sensor, data fusion, biased estimation, wind speed measurement.

Abstract. Multi-sensor data fusion techniques combine data from multiple sensors and related information to achieve more specific inferences compared with using a single, independent sensor. In order to make full use of the information collected by multi-sensor and to increase the accuracy of wind speed, a wind speed measurement method with data fusion based on biased estimation is proposed in this paper. Firstly, different kind of wind speed measurement methods is introduced. Secondly, biased measurement is presented based on ridge estimation, and a quantitative description method evaluating the reliability of the measurement data is proposed. The biased measurement reliability superior to the unbiased one is also verified. Finally, a huge amount of wind speed data illustrates that the presented method is effective.

\section{Introduction}

All The measurement of wind speed is a key element of power performance testing. There are many ways to measure the wind speed, such as cup anemometers, windcube and Meteorological mast. Therefore, the objective of the wind speed measurement procedure is to called data that meet a set of clearly defined criteria to ensure that the data is of sufficient quantity and quality to determine the power performance characteristic of the wind turbine accurately. With the purpose of reducing the uncertainties associated to the measurement processes, data fusion approaches are used on instrumentation and measurement. This will increase the confidence with which we can give results of measurements. The commonly used methods for the combination of information from quantitative data are based on algorithms that attribute weights to the available information.

Multi-sensor data fusion is a new research field, using multiple sensors for a particular problem about a study of data processing [1]. Multi-sensor data fusion technology is a strong practicality application technology in recent years, it is a new technology of multidisciplinary cross, involves signal processing, probability and statistics, information theory, pattern recognition, artificial intelligence and fuzzy mathematics theory[2]. The basic principle of multi-sensor data fusion technology is to make full use of multiple sensors resource like human brain integrated processing [3 4].

The purpose of this paper is to provide a procedure that will ensure consistency, accuracy and reproducibility into the wind speed measurement. A wind speed measurement method with data fusion based on biased estimation is proposed in this paper. The biased measurement method is presented based on multi-sensor, such as meteorological mast under test, windcub, nacelle anemometer and wind farm meteorological mast. A quantitative description method is proposed to evaluate the reliability of the measurement data. A scale experiment wind speed data illustrates that the proposed method is effective. 


\section{Wind speed measurement sensors}

\section{Windcube}

As a new generation of wind measurement device, windcube plays a more and more important role in the wind measurement field. Windcube will become one of the main ways of wind measurement. Based on the principle of laser pulse Doppler shift measurement, it can obtain available wind speed, wind direction, temperature, humidity, atmospheric pressure, turbulence and wind shear. It can be seen as a portable wind mast. There are no rotating parts and consumable in windcube, this can largely reduce the equipment failure rate and extend service life. The measurement result is not affected by cloud and mist, so it does not need to use cloud correction software.

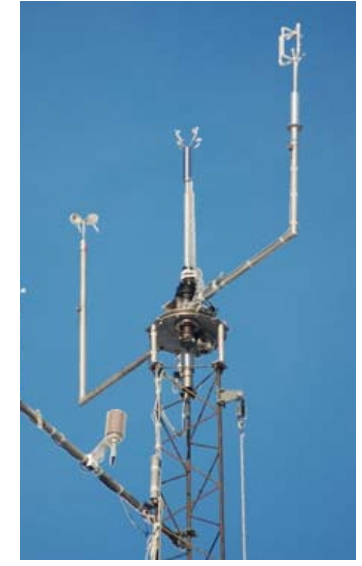

\section{Cup anemometer}

The sensing element of cup anemometer consists of three cup components made up of three carbon fiber wind cup and cup frame. The converter is tooth rotor and slit light coupling. When the cup rotates by horizontal wind, the shaft rotor rotation in the slit light coupling and the output signal is frequency. The converter of the sensors can be used precision conductive plastic potentiometer, thus voltage signal output changes on the potentiometer activities end.

\section{Meteorological mast}

Meteorological mast is used to observe and record the surface airflow movement. It is installed in the wind farm site with the truss frame structure or the cylindrical structure strengthened with steel wire suspension. Its height generally is $10-150$ meters. The anemometer, vane and monitoring equipment is installed at different height of mast, such as temperature, air pressure sensors. It can be all-weather continuous observation at wind condition site. The wind load of mast is little, it also have strong ability to resist wind. The mast is made small to collect data accurately, and minimize the gap between measured data and actual data.

\section{Measurement reliability analysis of biased estimation}

Assume that the sensor measurement model is as formula (1), the input noise w meet same Gaussian distribution, and do not related with unknown under test vector $x, \mathrm{E}\left(w^{\mathrm{T}} x\right)=0$ and w $N\left(0, \sigma^{2} I\right)$.

$$
y=H x+w
$$

To study the problems, the linear model $y=H x+w$ is considered. $\lambda_{1}, \lambda_{2}, \cdots \lambda_{n}$ is the characteristic root of symmetric matrix $H^{\mathrm{T}} H$, and meet $\lambda_{1} \geq \lambda_{2} \geq \cdots \geq \lambda_{n}>0 . \rho_{1}, \rho_{2}, \cdots, \rho_{n}$ is the standardization characteristic vector of characteristic root, Assume $G=(\rho 1, \rho 2, \cdots, \rho n) Z=H G, \Lambda=\operatorname{diag}\left\{\lambda_{i}\right\}, i=1,2, \cdots, n$ and $\alpha=G^{\mathrm{T}} x$, then the linear model can be converted into standard form, expressed as:

$$
y=Z \alpha+w
$$

The least squares estimate of the unknown vector is: 


$$
\hat{\alpha}_{\mathrm{LS}}=\Lambda^{-1} Z^{\mathrm{T}} y
$$

$\hat{\alpha}_{\mathrm{LS}}$ and $\hat{x}_{\mathrm{LS}}$ meet:

$$
\hat{x}_{\mathrm{LS}}=G \hat{\alpha}_{\mathrm{LS}}
$$

According to assumptions measurement vector y satisfy the Gaussian distribution, variance is $\operatorname{var}(y) \quad N\left(0, \sigma^{2} I\right)$. According to the formula (3), $\hat{\alpha}_{\mathrm{LS}}$ is linear transformation. as the formula (4), $\hat{x}_{\mathrm{LS}}$ is a linear transformation of $\hat{\alpha}_{\mathrm{LS}}$, so $\hat{x}_{\mathrm{LS}}$ satisfy the Gaussian distribution. $H=[1,1, \cdots, 1]^{\mathrm{T}}$, we can obtain the unbiased measurement value is

$$
\hat{x}_{\mathrm{LS}}^{i}=\sum_{j=1}^{n} \rho_{i j} \alpha_{\mathrm{LS}}^{j}, \quad i=1,2, \cdots, n
$$

Where, $\rho_{i j}$ and $\hat{\alpha}_{\mathrm{LS}}^{j}$ is the $j$-th element of $\rho_{i}$ and $\hat{\alpha}_{\mathrm{LS}}$. The estimated variance matrix of $\hat{x}_{\mathrm{LS}}$ is defined:

$$
\operatorname{var}\left(\hat{x}_{\mathrm{LS}}\right)=\sigma^{2}\left(H^{\mathrm{T}} H\right)^{-1}
$$

Ridge estimation has been widely used to improve the biased estimation method of least squares estimation variance. Because the measurement between unbiased and least squares estimate are equivalent, so this paper improved unbiased variance of measured data by introduce a small deviation, and it is called biased measurement process. Based on the model (1), ridge estimate is:

$$
\hat{x}_{\mathrm{RE}}=\left(H^{\mathrm{T}} H+k I\right)^{-1} H^{\mathrm{T}} y
$$

Ridge estimate can simply be defined:

$$
\hat{\alpha}_{\mathrm{RE}}=(\Lambda+k I)^{-1} Z^{\mathrm{T}} y=(\Lambda+k I)^{-1} \Lambda \hat{\alpha}_{\mathrm{LS}}
$$

Where, $k \geq 0$ is partial parameter. This formula (7) and (8) are:

$$
\begin{gathered}
\hat{x}_{\mathrm{GRE}}=\left(H^{\mathrm{T}} H+G^{\mathrm{T}} K G\right)^{-1} H^{\mathrm{T}} y \\
\hat{\alpha}_{\mathrm{GRE}}=(\Lambda+K)^{-1} \Lambda \hat{\alpha}_{\mathrm{LS}}
\end{gathered}
$$

When the value matrix $K$ of the diagonal elements is the same, the generalized ridge estimators convert to ridge estimation. The bias and variance are:

$$
\begin{gathered}
\operatorname{bias}\left(\hat{\alpha}_{\mathrm{GRE}}\right)=K(\Lambda+K)^{-1} \alpha \\
\operatorname{var}\left(\hat{\alpha}_{\mathrm{GRE}}\right)=\sigma^{2}(\Lambda+K)^{-1} \Lambda(\Lambda+K)^{-\mathrm{T}}
\end{gathered}
$$

Analysis (8) with (10) can be seen that ridge estimate $\hat{\alpha}_{\mathrm{RE}}$ and generalized ridge estimators $\hat{\alpha}_{\mathrm{GRE}}$ are least squares estimation of linear transformation, the transformation matrix consist of $(\Lambda+k I)^{-1} \Lambda$ and $(\Lambda+K)^{-1} \Lambda$. According to formula (5) and (10), the generalized ridge estimate available can described as:

$$
\hat{x}_{\mathrm{GRE}}^{i}=\sum_{j=1}^{n} \rho_{i j} \hat{\alpha}_{\mathrm{GRE}}^{j}=\sum_{j=1}^{n} \frac{\rho_{i j} \lambda_{j}}{\lambda_{j}+k_{j}} \hat{\alpha}_{\mathrm{LS}}^{j}
$$

Take $H=[1,1, \cdots, 1]^{\mathrm{T}}$, we can get the biased measurements of the unknown parameters according to the formula (13). $\hat{x}_{\mathrm{GRE}}$ is a linear transformation of $\hat{\alpha}_{\mathrm{GRE}}$, due to the linear combination of the normal distribution is still a normal distribution, at the same time, the generalized ridge estimators is also normal distribution.

$$
\operatorname{var}\left(\hat{\alpha}_{\mathrm{GRE}}^{i}\right)=\sigma^{2} \frac{\lambda_{i}}{\left(\lambda_{i}+k_{i}\right)^{2}}
$$

$\alpha_{\mathrm{GRE}}^{i}$ meet the normal distribution, probability density function of $\alpha_{i}$ is:

$$
f_{\mathrm{GRE}}\left(\alpha_{i}\right)=\frac{\lambda_{i}+k_{i}}{\sqrt{2 \pi \lambda_{i} \sigma^{2}}} \exp \left[-\frac{\left(\lambda_{i}+k_{i}\right)^{2}}{2 \lambda_{i} \sigma^{2}}\left(\alpha_{i}-\hat{\alpha}_{\mathrm{GRE}}^{i}\right)^{2}\right]
$$


Normally, when researchers dealt with unknown system deviation of measurement data, they tried to adopt a hypothesis model to describe the system deviation, and then eliminate or reduce the system deviation. Deviation on the basis of unbiased measurement was introduced. But the deviation which is introduced in this paper is known deterministic deviation, which is different from the usual unknown system error.

\section{Multi-sensor data fusion method based on biased estimation}

Multi-sensor Data Fusion ( MSDF) is an actually function of the human brain for the integrated treatment of complex issues imitation. MSDF takes advantage of multiple sensors. Through the multi-sensor and its reasonable control and use of observational information, the plurality of sensors redundant or complementary information in space or time based on certain criteria will be combined to obtain the test consistency description of the object, so that the information system thus obtained system better performance than a subset of its various components of the composition.

To illustrate the core problem, we use a combination of fusion convex structure to measure the same variety using biased estimate unknown parameters and measurement reliability data fusion method. Multi-sensor measuring system consists of $\mathrm{N}$ sensors, and assumed to have been obtain a single sensor is biased measurements, $\hat{x}_{i}=1,2, \cdots N$, So multi-sensor data fusion biased estimate is expressed as:

$$
\hat{x}_{F}=W_{1} \hat{x}_{1}+W_{2} \hat{x}_{2}+\cdots+W_{N} \hat{x}_{N}
$$

Convex combination coefficients satisfied:

$$
\sum_{i=1}^{N} W_{i}=1 \quad 0 \leq W_{i} \leq 1
$$

Multi-sensor data fusion biased estimate of variance and bias are analysis based on the formula (16) and (17), Since

$$
\operatorname{bias}\left(\hat{x}_{i}\right)=\mathrm{E} \hat{x}_{i}-x \quad i=1,2, \cdots N
$$

The bias of multi-sensor data fusion biased estimation is a linear combination of biased estimation error of each sensor. $\operatorname{bias}\left(\hat{x}_{i}\right)=\operatorname{bias}\left(\hat{x}_{j}\right) \quad i, j=1,2, \cdots N, i \neq j$

$$
\begin{aligned}
\operatorname{bias}\left(\hat{x}_{F}\right) & =\left(W_{1}+W_{2}+\cdots W_{N}\right)\left(\mathrm{E} \hat{x}_{i}-x\right) \\
& =\operatorname{E} \hat{x}_{i}-x=\operatorname{bias}\left(\hat{x}_{i}\right)
\end{aligned}
$$

Formula (20) described the estimated bias of many same sensor biased estimate fusion.

Assuming the measurement system consists of $\mathrm{N}$ identical sensors and biased measurement error is not related to each sensor, so the mean square error of multi-sensor data fusion is:

$$
\operatorname{MSE}\left(\hat{x}_{F}\right)=\frac{\mathrm{var}}{N}+\operatorname{bias}^{2}
$$

Where, var and bias are variance and bias of single sensor biased estimation.

Because they are not related to different partial measurement error of the sensor, $\operatorname{var}\left(\hat{x}_{i}\right)=\mathrm{E}\left(\hat{x}_{i}-\mathrm{E} \hat{x}_{i}\right)^{2}$ and $\operatorname{bias}^{2}\left(\hat{x}_{i}\right)=\left(\mathrm{E} \hat{x}_{i}-x\right)^{2}, i=1,2, \cdots N$. Formula (21) is:

$$
\begin{aligned}
\operatorname{MSE}\left(\hat{x}_{F}\right)= & \mathrm{E}\left[\sum_{i=1}^{N} W_{i}^{2}\left(\hat{x}_{i}-\mathrm{E} \hat{x}_{i}\right)^{2}+\sum_{i=1}^{N} W_{i}^{2}\left(\mathrm{E} \hat{x}_{i}-x\right)^{2}\right] \\
& +\sum_{i=1}^{N} \sum_{j=i+1}^{N} W_{i} W_{j}\left(\mathrm{E} \hat{x}_{i}-x\right)\left(\mathrm{E} \hat{x}_{j}-x\right) \\
= & \sum_{i=1}^{N} W_{i}^{2} \operatorname{var}\left(\hat{x}_{i}\right)+\sum_{i=1}^{N} W_{i}^{2} \operatorname{bias}^{2}\left(\hat{x}_{i}\right) \\
& +\sum_{i=1}^{N} \sum_{j=i+1}^{N} W_{i} W_{j}\left(\mathrm{E} \hat{x}_{i}-x\right)\left(\mathrm{E} \hat{x}_{j}-x\right)
\end{aligned}
$$


Convex combination biased estimates data fusion can be transformed to the following optimization problem, namely to find the optimal parameters of a convex combination of $W_{i}$, $i=1,2, \cdots N$, to minimize the mean square error.

$\min \operatorname{MSE}\left(\hat{x}_{F}\right)$

$$
\text { s.t. } \sum_{i=1}^{N} W_{i}=1 \quad 0 \leq W_{i} \leq 1
$$

The measuring system is composed by a plurality of identical sensors.

$\operatorname{bias}^{2}\left(\hat{x}_{i}\right)=\operatorname{bias}^{2}\left(\hat{x}_{j}\right)=\operatorname{bias}^{2}$ and $\operatorname{MSE}\left(\hat{x}_{i}\right)=\operatorname{MSE}\left(\hat{x}_{j}\right)=\operatorname{MSE}$, formula (23) can be simply described as:

$$
\begin{aligned}
\min \operatorname{MSE}\left(\hat{x}_{F}\right)= & \operatorname{MSE} \sum_{i=1}^{N} W_{i}^{2} \\
& +\operatorname{bias}_{i, j}^{2} \sum_{i=1}^{N} \sum_{j=i+1}^{N} W_{i} W_{j}
\end{aligned}
$$

The extremum of the objective function $\operatorname{MSE}\left(\hat{x}_{F}\right)$ is:

$$
\begin{aligned}
\operatorname{MSE}_{O p t}\left(\hat{x}_{F}\right)=\frac{\operatorname{MSE}}{N}+\frac{(N-1) \operatorname{bias}_{i, j}^{2}}{N} \text { When } N & =2 \text {, mean square error reduction is: } \\
\operatorname{MSE}\left(\hat{x}_{F}\right) & =\frac{\operatorname{MSE}}{N}+\frac{(N-1) \operatorname{bias}_{i, j}^{2}}{N} \\
& =\frac{\operatorname{var}+\operatorname{bias}^{2}}{N}+\frac{(N-1) \operatorname{bias}_{i, j}^{2}}{N} \\
& =\frac{\operatorname{var}}{N}+\operatorname{bias}^{2}
\end{aligned}
$$

The variance is:

$$
\operatorname{var}\left(\hat{x}_{F}\right)=\frac{\operatorname{var}}{N}
$$

Especially, when $N=1$, formula (25) is the Mean square error of one sensor.

\section{Data analysis and result}

The data fusion algorithm is verified by the measured wind speed as the original values in a wind farm, the sampling is the actual wind speed and meteorological data per hour in the whole year of 2014 . According to different seasonal characteristics of wind speed, the forecasting model should be different. This paper selects the region is a typical day of December, to analyze the sample data modeling.

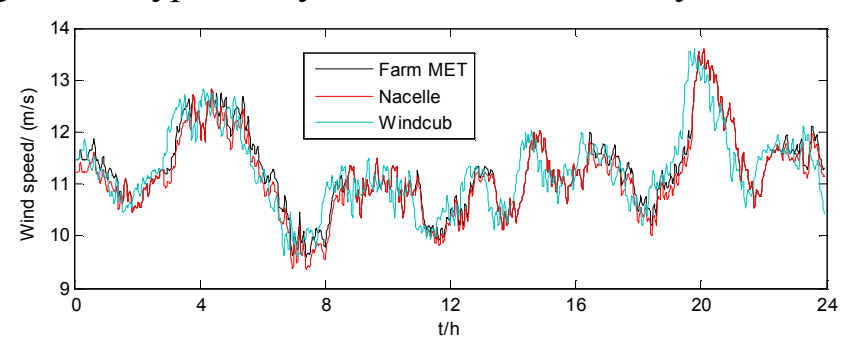

Fig 2. Wind speed curves of different measuring sensors

Fig 2 shows the wind speed curves among windcub, nacelle anemometer and wind farm meteorological mast. The meteorological mast under test is chosen as the standard wind measurement facility. The data of wind speed measured by standard MET mast is shown in Fig 3. 


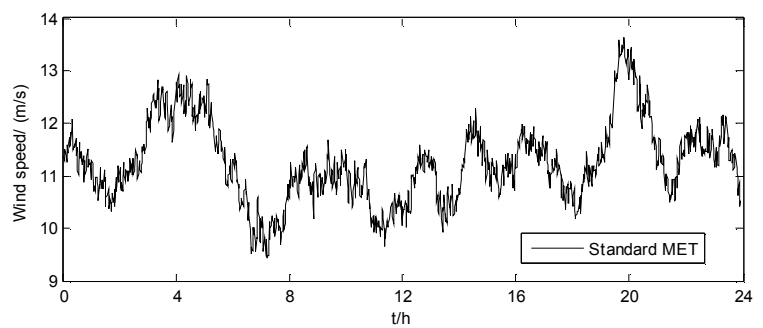

Fig 3. Wind speed curve of standard MET mast

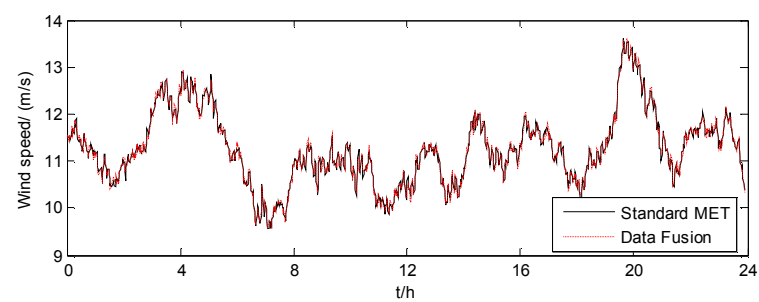

Fig 4. Comparison of wind speed based on data fusion vs. standard MET mast

The comparison between direct measurement and calculation result using the proposed indirect estimated method can be seen in the Fig.4. The wind speed curve based on data fusion appears to be similar to the curve based on standard MET mast. These results differ by approximately $0.5 \%$.

\section{Conclusion}

The paper presents a wind speed measurement method with data fusion based on biased estimation. Biased measurement is presented based on ridge estimation, and a quantitative description method evaluates the reliability of the measurement data. The method makes full use of the information collected by multi-sensor and increases the accuracy of wind speed measurement. The experiment wind speed data illustrates that the proposed method is effective.

\section{Acknowledgment}

This work was financially supported by the National Natural Science Foundation of China (51207095), scientific and Technological Projects of Liaoning Province (2011220017), Scientific and Technological Projects of Liaoning Province (2012220042).

\section{References}

[1] H.C.Yan, X.H.Huang and M Wang, Muti-sensor data fusion technique and its application, Journal of Transducer Technology, vol. 24, pp. 1-4, 2005.

[2] M.G.Huang, S.C.Fan, D.Z.Zheng and W.W.Xing, Research progress of multi-sensor data fusion technology, Transducer and Microsystem Technologies, vol. 29, pp. 5-12, 2010.

[3] R Luo, $\mathrm{H} \mathrm{Xu}$ and T Tian, Complex system multisensor data fusion technology and its applications research, China Measurment Technology, vol. 32, pp. 17-21, July 2006

[4] Y.L.Yue, X.Zuo and X.L.Luo, Improving Measurement Reliability with Biased Estimation for Multi-sensor Data Fusion, ACTA Automation Sinica, vol. 40, pp. 1843-1851, September, 2014 